\title{
Comment on: Relation of blood pressure variability to left ventricular function and arterial stiffness in hypertensive patients
}

Singapore Med J 2021; 62(11): 616 https://doi.org/10.11622/smedj.2021228

Dear Sir,

We read with great interest the article by Shin et $\mathrm{al}^{\left({ }^{(1)}\right.}$ in which the authors studied patients with hypertension and found an independent correlation between diurnal systolic blood pressure (BP) variability and three-dimensional left ventricular area strain, which could serve as a new tool for evaluating subclinical systolic function through speckle-tracking echocardiography.

We recently observed a similar result in a small population of patients with long-lasting Parkinson's disease (PD) without hypertension who showed wide fluctuations in their daily BP due to autonomic dysfunction. ${ }^{(2)}$ In these patients, 24-hour systolic BP variability was correlated with two-dimensional global longitudinal strain and weakly related to other indices of left ventricular function. Remarkably, these patients with PD without hypertension shared several echocardiographic similarities with a control group of patients with hypertension, including left ventricular mass index and left atrial size. We think that the findings of these two studies, together with previous data obtained from normotensive patients with Type 1 diabetes mellitus, ${ }^{(3)}$ strengthen the hypothesis that BP variability may, in itself, be a cause of left ventricular damage and remodelling independent of BP values. Thus, the study by Shin at $\mathrm{al}^{(1)}$ and our study provide the first evidence that speckle-tracking echocardiography techniques could serve as useful tools for detecting very early signs of subclinical BP variability-related systolic dysfunction.

Yours sincerely,

Giuseppe $\underline{\text { Caminiti }^{1}}$, Iellamo $\underline{\text { Ferdinando }}^{2}$, Rosalba $\underline{\text { Massaro }}^{1}$, Maurizio $\underline{\text { Volterrani }}^{1}$

${ }^{1}$ Cardiology Rehabilitation Unit, S Raffaele IRCCS, 'Dipartimento di Scienze Cliniche e Medicina Traslazionale, Università Tor Vergata, Roma, Italy. giuseppe.caminiti@sanraffaele.it

\section{References}

1. Shin SH, Jang JH, Baek YS, et al. Relation of blood pressure variability to left ventricular function and arterial stiffness in hypertensive patients. Singapore Med J 2019; 60:427-31.

2. Caminiti G, D'Antoni V, Morsella V, et al. Correlation between systolic blood pressure variability and global longitudinal strain in patients with Parkinson's disease and dysautonomia. J Cardiovasc Dis Res 2020; 11:1-3.

3. Taskiran M, Rasmussen V, Rasmussen B, et al. Left ventricular dysfunction in normotensive Type 1 diabetic patients: the impact of autonomic neuropathy. Diabet Med $2004 ; 21: 524-30$.

Editor's note: The authors, Shin et al, have declined to respond to the above letter. 